\title{
A New Method for Positioning Persian License Plates
}

\author{
Mehdi Rahmanian, Reza Javidan, Member, IACSIT, and Manijeh Keshtgary
}

\begin{abstract}
Today the necessity of using smart systems which are able to diagnose the cars plague correctly from pictures is felt more than ever by expanding the number of vehicles. In License Plate Recognition systems, detecting plaque location is considered the most important phase so that the correct rate of it, will affect all subsequent phases. There are different images with different resolutions and sever changes in the size of images which taken through being far or near to the camera, it makes many existing methods face problems or longer processing. In this paper a new method is suggested based on simulation concepts which used in spectra analysis for accurate and fast position detection by using edge concepts and morphological operators in image processing. The suggested method is a compound method and fast enough and it extracts the plague location from vehicle image correctly. The algorithm is implemented in MATLAB and the results obtained agreed with theoretical predictions. The proposed method has $93.3 \%$ of correct detection rate and $150 \mathrm{~ms}$ of processing time.
\end{abstract}

Index Terms-LPR, Edge detection, Morphological operators, Spectral analysis.

\section{INTRODUCTION}

Intelligent Transportation Systems (ITSs) have many interactions with the people lives. On the other hand, License Plate Recognition systems (LPRS) play a major role in automatic monitoring of traffic rules and maintaining law enforcement on public roads. LPRSs are considered as a branch of machine vision that they extract and read plague by using of image processing. In addition, these systems are used in several other cases, such as identifying stolen vehicles and car racing. These systems usually include a digital camera, a software module which is used connecting the camera to the License plate recognition software, and detecting, extracting software. The camera prepares images by using a predetermined quality and passes them to software modules.

Plate recognition systems perform this task using three steps including finding location of plate in the vehicle image; plate segmentation; and finally character recognition. The plate region extraction is the most challenging part of the entire system and different method has been proposed for it. License plate positioning quality has influence on the accuracy of license plate recognition. The algorithms which are used to identify the license plate location can generally be divided into four general categories: methods based on classification, methods based on edge detection, methods based on morphological operators, and methods based on spectral analysis. The most popular method which is

Manuscript received September 23, 2011; revised October 31, 2011.

All Authors are with Department of Computer Engineering and Information Technology, Shiraz University of Technology, Shiraz, Iran (e-mail: mehdirahmanian@ymail.com; reza.javidan@gmail.com; keshtgari @sutech.ac.ir). considered in the classifier is usage of neural networks. It is usually used a three layers neural network to do it. The first layer is used as input layer, second layer as hidden layer and the third layer as the output layer [5]. In such methods the input layer includes a collection of license plate features.

Histogram analysis of images is also sometimes used. License plate location in images due to having many characters and their repeating in sequence usually contains a higher histogram than other areas [12]. Using the histogram analysis is not a suitable criterion and there is this probability that there are other areas similar to it [5]. In [6], a six-layer cascaded classifier is constructed to increase the detection speed, in which the first two layers are based on global features and the last four layers are based on local Haar-like features.

In [7], a hierarchical algorithm for practical license plate recognition is proposed. This method is designed especially for Farsi plate detection. For plate location process, first the vehicle image is preprocessed. Then resulting is converted to the binary image Then Morphological closing and opening operations. Then noisy object is removed. Finally, candidate plate is obtained by using connected object. In [8], a novel approach of license plate recognition system is proposed by adopting comprehensive feature of license plate. It uses linear fitting method and projecting method to rectify the position of license plate. Then the color set of license plate are combined to match features and stroke width constraint to locating the license plate.

Using edges alone will not be able to detect license plate location and the reason for this is that, detecting edge operators don't only deal to extract the edge of license plate and they extract all edges which are on the image, that also including backgrounds. The advantage of edge detection methods are their high speed. Using the methods, based on image scanning require to check the image pixel by pixel that it's a time consuming method and in spite of that is accurate [3],[4]. Using the spectral analysis method is also a very accurate method which requires complex mathematical calculation and it will be time consuming.

The methods based on histogram analysis are also not suitable for images that contain noise and are unclear and they are usually used as a combination with another method. Using methods based on morphological operator are not suitable for real time systems, but they have a high accuracy.

In this paper, all input images convert into a typical form by using a set of image processing operators. The target form in this study is removing color effect, making input images in one size, removing noises by using a specified threshold. After standardization of input images to the defined concepts and fundamental principles in spectral analysis, the edges of the images are processed. After simulating spectral analysis, the license plate location is detected with a high probability form the picture and the desired part of license plate is cut 
from car image and as an input for next stage. Afterward, morphological operators are used to develop and expand the likely location and check and extract an object with specific features and appropriate Iranian license plate.

The remaining of the paper is organized as follows: in Section II the proposed method for positioning Persian plates is presented. Experimental results are outlined in Section III. Finally conclusions and remarks are presented in Section IV.

\section{The Proposed Method}

In Fig. 1 the entire proposed algorithm is shown. In this algorithm the input images are captured by using a digital camera capable of capturing images at high resolution. The first part of proposed algorithm is preprocessing the captured image because of complex background and uncertain factors in license plate due to weather condition, illumination, fouling and occlusion of license plate.

The second part of proposed algorithm is detecting edges of preprocessed image. Detecting image's edge has different method that we will explain later.

The third and fourth part of proposed algorithm is detecting and extracting the probable location of license plate on the image's edge to the defined concepts and fundamental principles in spectral analysis. For searching the probable location of license plate on the image we have proposed a new fast method that we will explain.

In the fifth part of proposed algorithm we apply morphological operations with suitable structural element to the output image of last section to extract the connected components on the image.

The last part of proposed algorithm is extracting object with suitable condition.

\section{A. Pre-Processing}

The input images have different colors and various light rate .Working with colorful images is complex and difficult and they increase the processing time greatly. In this paper to remove color effect, all input images in any format, are made out from colorful mode and turned into gray scale. In this paper, first the image is made out from colorful mode and turned into gray mode. Through this way, the problem of working with different colors will be lost for doing this. The images are taken as input, usually have different size and their size is too large and makes the image processing functions face with problem. In addition, large images will be effective in processing time and processing speed. In this research in order to remove the effect of images size as one of the pre-processing actions, The size of images are reduced into a quarter of real value of the main images by helping of resizing functions in image processing and passed to next stages.

All images which taken don't have the same quality and they may be prepared in various conditions. For example, perhaps the weather conditions to be unsuitable and the image which taken to be unclear and blur or other factors that might make pictures associated with noise and distortion. In result, it is necessary before future processing, as far as possible make pictures transparent. There are various methods for removing noise and uneven in image processing.

One of the widely useful methods for removing noise is using of Gaussian filter. Most likely noises are removed significantly by applying it. If there are more noises, more filters should be applied on the image.

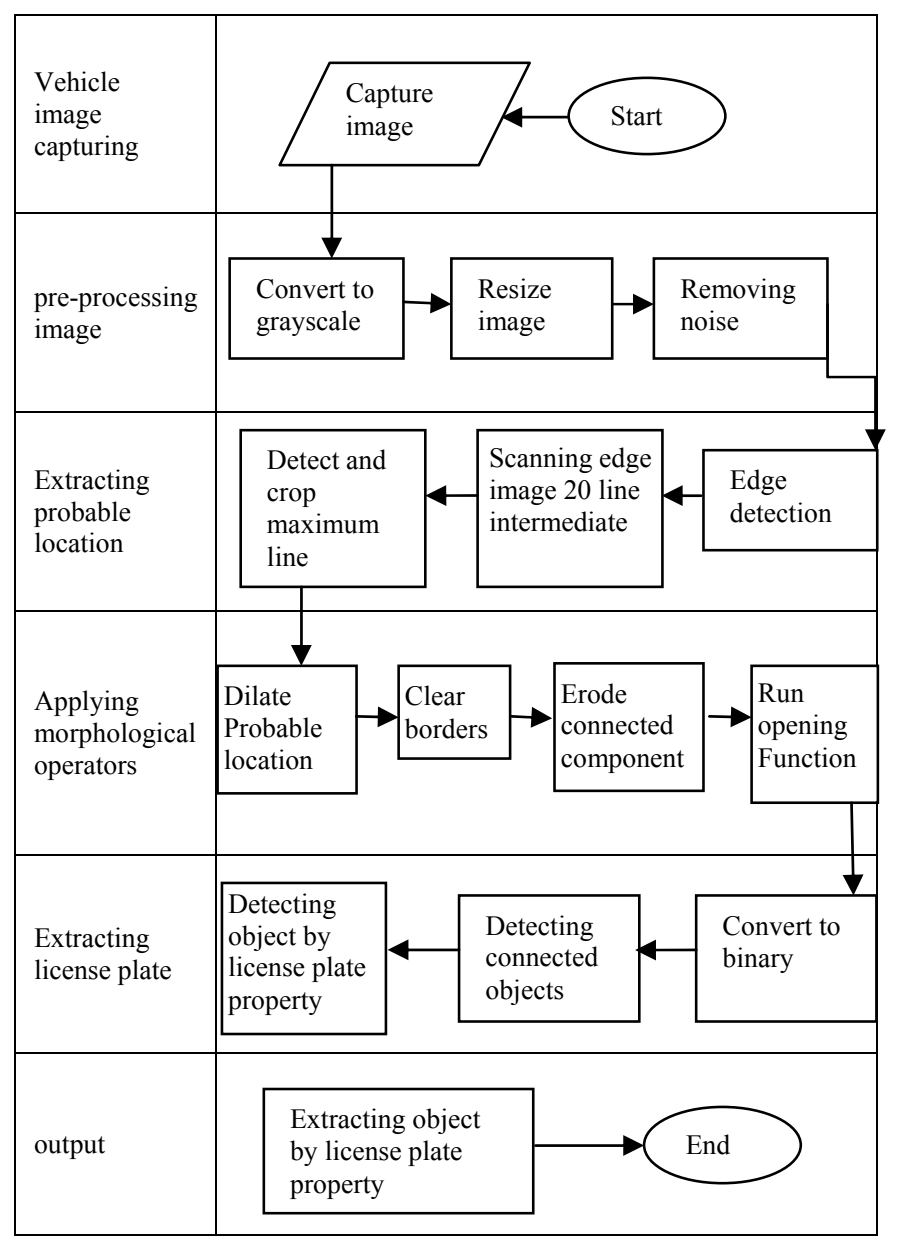

Fig. 1.The flowchart of proposed method

Another widely use of the filters for removing noise is using of Median filter. This filter is non linear and removes noise well .The following image shows an image which is applied the pre-processing actions on.

\section{B. Edges Extraction}

The idea that used in this study is derived from spectral analysis .concepts that used in spectral analysis, are analyzed and reviewed in next section completely and simulated by using of initiative method.

Before simulation of spectral analysis is necessary that the input image is made out from the original mode and turned into the edge image in order to simulating .Since spectral analysis significantly is related to light changes .The edge image is a very close concept to spectral analysis.

Edge is the most basic feature of image, so the edge indicates step change of the gray level on its surrounding pixels. License plate location on the image is a location that has maximal change of intensity because of presence of consecutive characters. This is one of the basic property that license plate region distinguishes from other region in the vehicles image.

Commonly used edge detection operators have Prewitt operator, SOBEL operator, canny operator, LOG operator, Robert operator and other operator. The gradients are derived by multiplying with the mask value for each pixel and its 
neighboring pixels. The experiment indicated that using the SOBEL edge detection operator can better stand out the edge characteristic of license plate, and speed is faster. The SOBEL operator uses two masks to find vertical and horizontal gradients. It is hard to separate the license plate region from the others in horizontal gradients image with, but it is easy to detect the license plate region from the vertical gradients image because the magnitude of vertical gradients is strong in the characters of license plate image. In our proposed method, we use SOBEL operator to filter the gray image and get vertical edge image.

The following image shows vertical mask action on a preprocessed image.

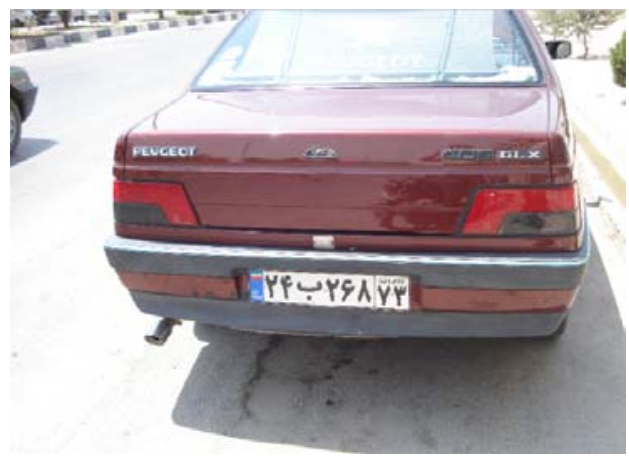

(a)

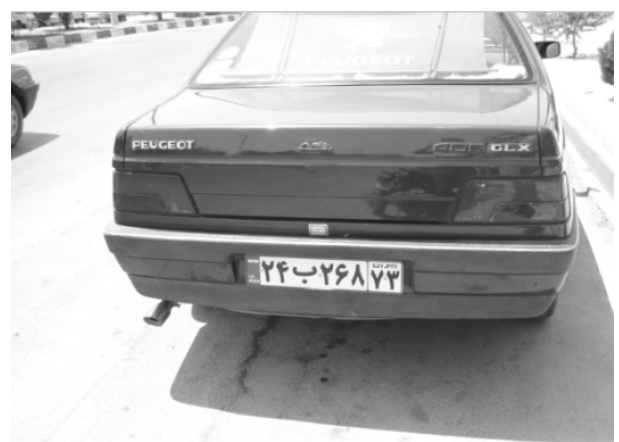

(b)

Fig. 2. (a) Input image, (b) Preprocessed image

\section{Spectral Analysis Simulation}

This part is the most important part of recommended method. In this section the approximate license plate location is detected precisely through spectral analysis simulation by using of edge.

By analyzing of new Persian license plate, it is found out that they have liner letters and characters and specific frequency in light intensity. This frequency is a good reason for using Fourier transform discrete. Fourier transform is calculated by using (1).

$$
\begin{aligned}
& F(u)=\frac{1}{M} \sum_{x=0}^{M-1} f(x) e^{\frac{-j 2 \pi u x}{M}} \\
& \text {, for } u=0,1, \ldots, M-1
\end{aligned}
$$

In many researches, Fourier analysis is used as like as above.

In study since Fourier transform is an unclear, to account this measure which is called spectrum density is used (2).

$$
\prod_{f}^{N}(u)=\frac{1}{N}\left|\sum_{x=0}^{N-1} f(x) e^{-j 2 \pi u x}\right|
$$

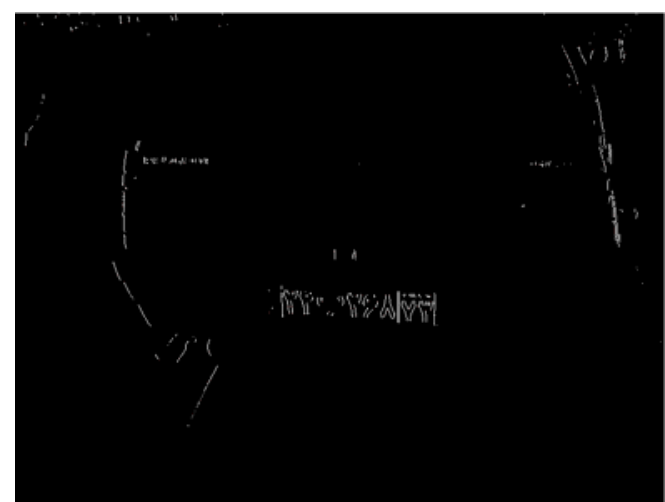

Fig. 3. Vertical SOBEL edge detection

Doing above processing on an image through spectral analysis, is required to use advanced mathematical functions and complex computes.

In this paper, similar to that is done in the image processing through spectral analysis some operations are done by using of edge concept and innovative processing on the image edge.

In the first section of spectral analysis it is told that in license plate location the frequency in light intensity is a good reason for Fourier transform using .In definition of edge is also presented that the edge is made through light changing in an image. Therefore, by getting the edge image, we have achieved the brightness changes.

In spectral analysis, calculating the transform is not just enough and it is necessary to calculate its amount. In this study, calculating of this amount is done by numbering edges of each row. The area of image which this density becomes maximum indicates the license plate location with a high probability. Checking of edge density is not done for all rows in this paper, but it is processed partially, in this way that instead of scanning all rows, it scans rows with a 20 distance from each other. Performing this action reduces processing greatly and increases the speed. The reason for choosing twenty numbers is that the rage width of new Iranian license plate is 40 pixels and maximum density occurs in the middle area of license plate.

Selecting appropriate number is an effective parameter in the accuracy of this method. The achieved number is obtained after review of numerous images.

In the following figure, the chart of edges of dense which are on the image rows, is shown, that equal to Fourier transform amount of each row.

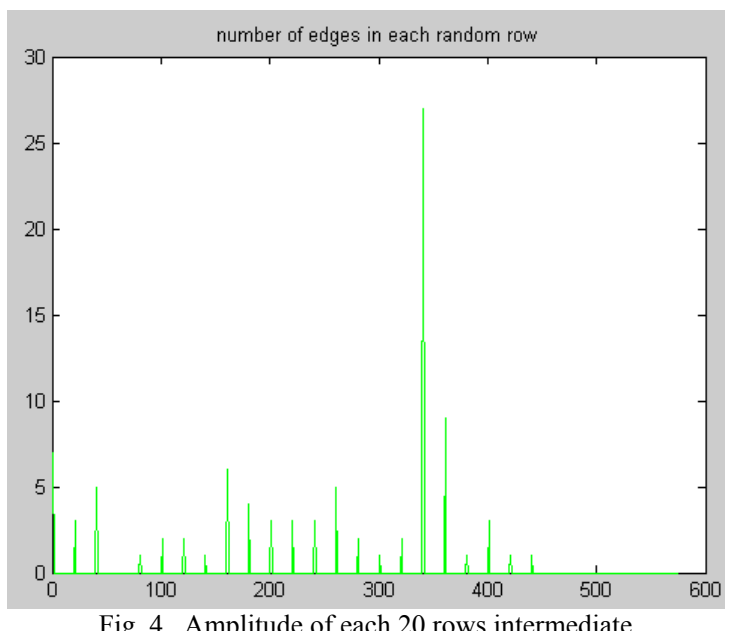

Fig. 4. Amplitude of each 20 rows intermediate 
In this figure, the horizontal axis indicates the number of rows and vertical axis indicates the number of edges in that row.

After being specified the probable license plate location, an area is extracted with 35 rows above and 35 rows below from the main image.

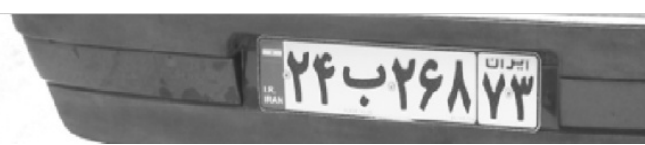

Fig. 5. Cropping the row with the max amplitude

The reason for choosing a number more than twenty at this stage is that the probable image of the license plate doesn't come on the side time of output image and doesn't effect on the next phases.

After determining the probable location next processing will be focused on this area. This courses processing time decrease significantly.

\section{Applying Morphological Operators}

Once the probable location of license plate has been cropped, the morphological operator is used to detect connected components of license plate.

Some methods use morphological operator on the whole image [3] Usually, morphological operators needs large time for processing, but in our proposed method because it is operated only on the probable license plate, it does not need more processing.

In the first step to connect the related component, morphological dilation operation with suitable structural element apply to the cropped gray scale image. The most important parameter in this step is selecting suitable structural element that can dilate enough and connect related component. To do this, we use two vertical and horizontal lines as structural element.

As the license plate is consist of characters written in black, we use IMFILL function to connect them to the connected component.

Fig. 6 shows filling image after applying dilation to the cropped image.

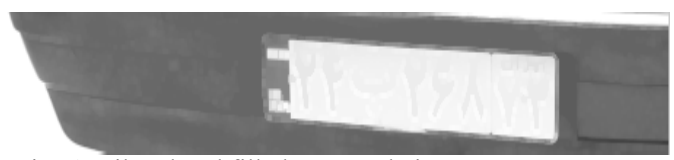

Fig. 6. Dilated and filled gray scale image

Some connected components are connected to the border that should be removed. Usually, license plate is located on the center of cropped image. The most important parameter in this part is the height of band of cropping after detecting the probable location of license plate. To solve this problem we can crop image with a bigger band. Another important parameter in this part is the angle of license plate that is related to the location of camera to the car.

Fig. 7 shows clear bordering image.

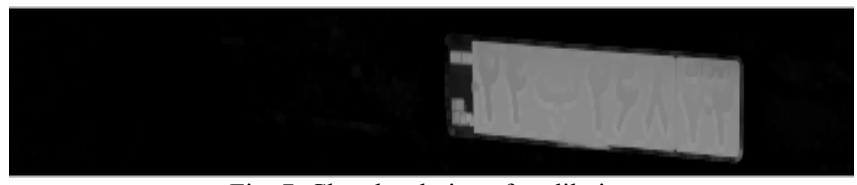

Fig. 7. Clear bordering after dilation
After clear bordering in order to make the connected components look natural, we smoothen the connected components by eroding the image twice with a suitable structural element. Fig. 8 shows eroded image.

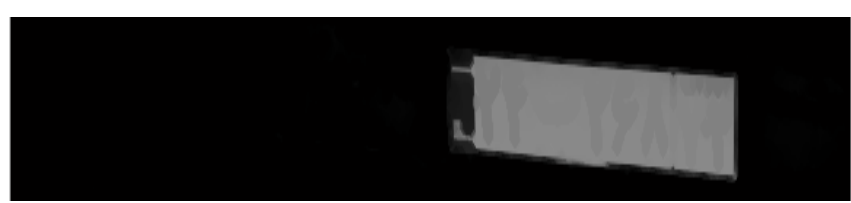

Fig. 8. Eroding connected component

Once the image has been converted to the connected component, the gray scale image is converted into its binary image by using suitable threshold. In this way we remove the effect of intensity and all of connected components are converted to the white object. Fig.9 shows binary image of last image.

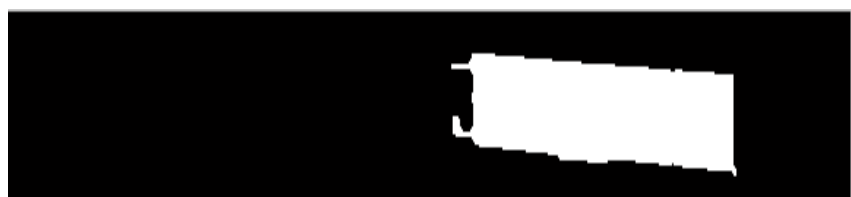

Fig. 9. Binary image of gray scale connected component

\section{E. Extracting an Object with License Plate Features}

After defining the connected components to detect the most possible license plate region from the probable region, the geometrical of the license plate are introduced to measure the possibility value. The following defines the geometrical features: 1) Area: If the candidate region is large, it is more likely being a license plate. 2) Orientation: The orientation of each possible license plate region can be measured. A license plate usually appears horizontally. There are many geometrical parameters that we can measure such as density. Measuring all of parameters needs more times and processing.

In this paper to reduce processing time we note two geometrical properties of license plate. After measuring geometrical parameters for each connected component we extract the connected component that is nearest to the license plate property.

The result of extracting connected component by the properties of license plate is shown in Fig. 10.

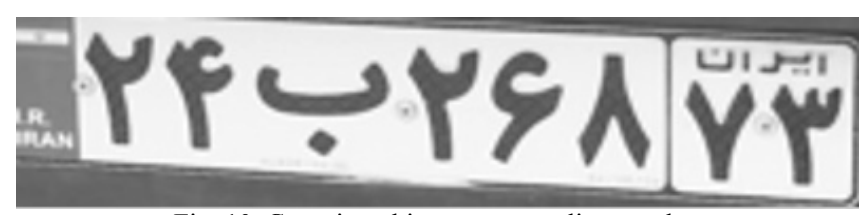

Fig. 10. Cropping object nearest to license plate

\section{EXPERIMENTAL RESULTS}

In our experiments, the proposed method was implemented in MATLAB. The dataset we used in this study include 150 vehicle which captured by a color digital camera in different angles and different hours of day. The distance between vehicles to camera when optimum frame was captured, is in range of 5 to $9 \mathrm{~m}$. All the pictures are captured from the rear end. Our experiments shows that plate positioning accuracy is about $93.3 \%$. The method is presented 
in this study is a fast and combination method and it doesn't depend on the angle of taking picture, and some parameters such as angle, color, and type of license plate in compare with some methods. The recommended algorithm is analyzed on a hundred vehicle image with different condition. The results express that if the images can be taken with a standard and certain distance, the amount of speed and accuracy of this method will be very acceptable.

Choosing an appropriate number in order to projecting image is so effective on accuracy of the method .Choosing a small number causes addition processing and choosing a large number leads to lose the probable location. The selected number in this study, is the result of reviewing of different images in different circumstanced.

The achieved results, by the recommended method similar to spectral analysis and with less processing, are obtained.

Applying morphological operators on images are usually time consuming. In this study, morphological operators are applied just on the probable area that it is so effective on the accelerating of method.

In our experiments, the proposed method was implemented on the image that is captured from the front of the car and the same result is obtained. The result of positioning Persian license plate on an image that is captured from the front is shown in Fig. 11.

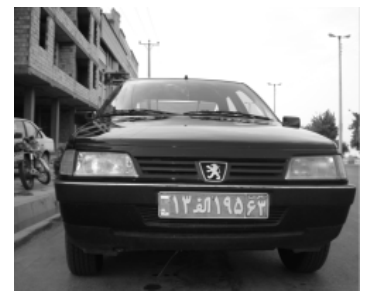

(a)

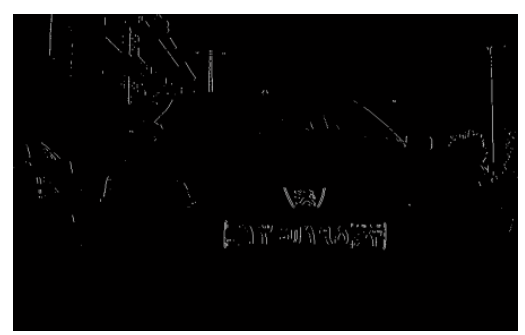

(c)

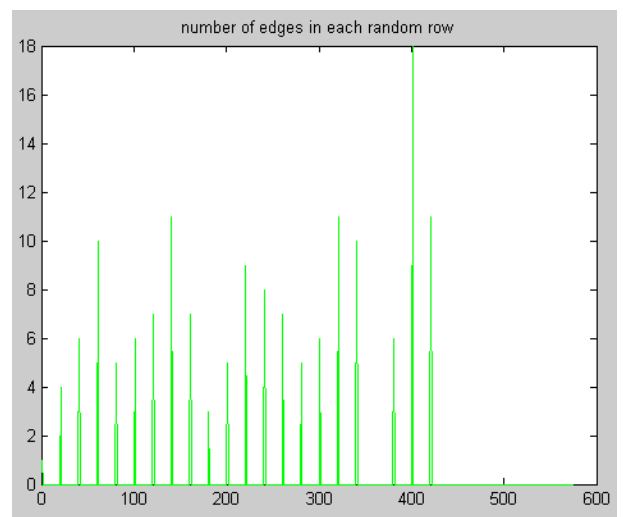

(d)

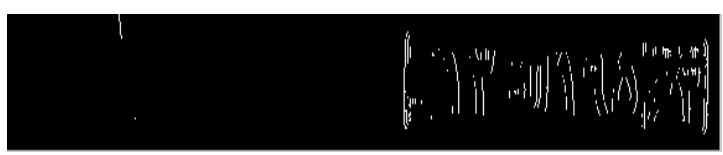

(e)

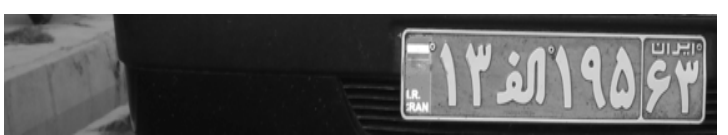

(f)

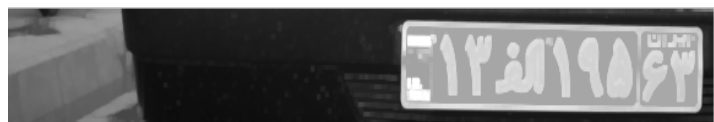

(g)

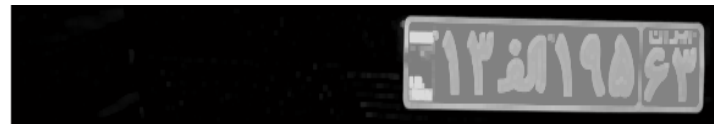

(h)

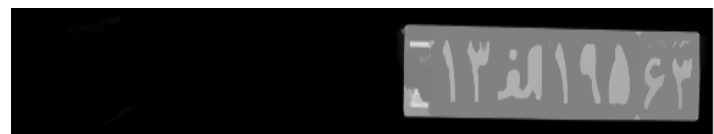

(I)

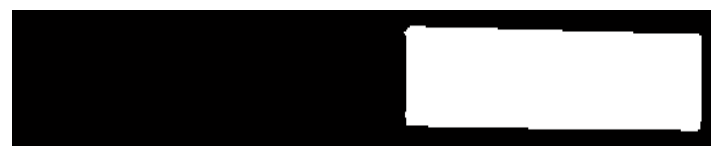

(j)

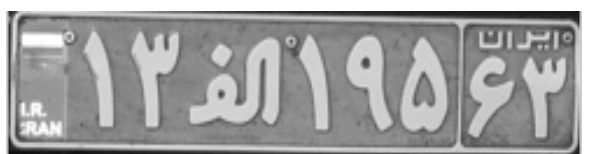

(k)

Fig. 9. (a)Input image, (b) Preprocessed image, (c) Edge extraction, (d) Number of edges in 20 intermediate rows, (e) Probable position of license plate, (f) Cropping probable location, (g) Dilating Probable location, (h) Clear bordering of dilated image, (I) Eroding probable location, (j) Binary image of dilated and eroded image, (k) Cropping object nearest to license plate

The result of implementing our proposed method on 150 images of database is shown on Table I.

TABLE I: THE RESUlT OF PROPOSED METHOD

\begin{tabular}{|c|c|c|c|c|}
\hline $\begin{array}{c}150 \text { images in } \\
\text { database }\end{array}$ & $\begin{array}{c}\text { Numbers of } \\
\text { correct } \\
\text { positioning }\end{array}$ & $\begin{array}{c}\text { Numbers of bigger } \\
\text { or smaller than } \\
\text { correct }\end{array}$ & $\begin{array}{c}\text { Numbers of } \\
\text { false } \\
\text { positioning }\end{array}$ & $\begin{array}{c}\text { Mean time } \\
\text { of } \\
\text { processing }\end{array}$ \\
\hline $\begin{array}{c}\text { Numbers of } \\
\text { image }\end{array}$ & 140 & 4 & 6 & $150 \mathrm{~ms}$ \\
\hline Percent & $93.3 \%$ & $2.6 \%$ & $4 \%$ & \multicolumn{1}{|c}{} \\
\cline { 1 - 3 } & \multicolumn{4}{|c}{}
\end{tabular}

\section{CONCLUSION}

Using spectral analysis is one of the most popular and accurate methods, in detecting plague, but the amount of processing and mathematical counts of this method is high and processing time, too.

In this paper we propose an efficient method which is capable of detecting plate in the vehicle 's image which is taken from the rear end by using the concept of edges and morphological operators. We have successfully designed and implemented the key element of license plate recognition systems, license plate positioning. The main target of paper was detecting the probable location of license plate to reduce the time of processing. This goal has been accomplished in this paper.

The recommended method applies the embedded features in edge image of license plate very well and produces such a 
result which is in the spectral analysis. The recommended method applies the morphological operators well in order to identifying the license plate very early just by applying on probable area.

In fact, the presented method is a combination of simulating spectral analysis for detecting probable plague location and morphological operators for identifying plague object.

\section{REFERENCES}

[1] Amir Azim Sharifloo, Mehrnoush Shamsfard "A Bottom up Approach to Persian Stemming", Shahid Beheshti University, Tehran, Iran.

[2] C. Peters, V. Jijkoun, T. Mandl, H. M"uller, D. Oard, A. Pe ̃nas, and D. Santos, Eds., "Advances in Multilingual and Multimodal Information Retrieval", Lecture Notes in Computer Science. Berlin: Springer-Verlag, Vol. 5152, 2008.

[3] Mehdi Rahmanian, Reza Javidan, Mashallah Abbasi Dezfuli, "Efficient Parameters in Farsi License Plate Positioning by Using Edges and Morphological Operator", Canadian Journal on Image Processing and Computer Vision, Vol. 2 No. 6, July 2011

[4] Mehdi Rahmanian, Reza Javidan, Mashallah Abbasi Dezfuli, "A Fast and Hybrid Method for Persian License Plate Positioning Base on Edges and Morphological Operator", Islamic Azad University, Neyriz, Iran.

[5] Delforouzi A., Pooyan M., "Efficient Farsi license plate recognition" In proceeding of $7^{\text {th }}$ IEEE International Conference on Information, Communications and Signal Processing, 2009.

[6] Chengpu Yu, Mei Xie, Jin Qi, "A Novel System Design of License Plate Recognition" in proceeding of IEEE International Symposium on Computational Intelligence and Design, 2008.

[7] Megalingam Rajesh Kannan, Krishna Prasanth, Somarajan Pratheesh, Pillai, Vishnu A., UlHakkim Reswan, "Extraction of license plate region in Automatic License Plate Recognition" in proceeding of 2nd IEEE International Conference on Mechanical and Electrical Technology (ICMET), 2010.

[8] Lihong Zheng, Xiangjian He, Samali B., Yang L. T., “Accuracy Enhancement for License Plate Recognition" in proceeding of 10th IEEE International Conference on Computer and Information Technology (CIT), 2010.

[9] Yucheng Li, Yisong Liu, Mushu Wang, "Study and Realization for License Plate Recognition System", in proceeding of IEEE Asia-Pacific Conference on Information Processing, 2009.
[10] Shen-Zheng Wang and Hsi-Jian Lee, "Detection and recognition of license plate characters with different appearances", In proceeding of IEEE Intelligent Transportation Systems, 2003.

[11] Ching-Tang Hsieh, Liang-Chun Chang, Kuo-Ming Hung, Hsieh-Chang Huang, "A real-time mobile vehicle license plate detection and recognition for vehicle monitoring and management", in proceeding of IEEE Joint Conferences on Pervasive Computing (JCPC), 2009.

[12] Jianxia Wang, GuilongGao, Huili Yang, "Research and implementation of license plate location based on histogram division method" In proceeding of 9th IEEE International Conference on Electronic Measurement \& Instruments, 2009.

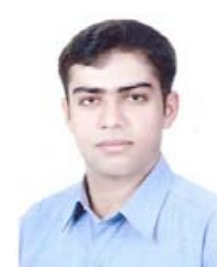

Mehdi Rahmanian received his undergraduate degree in Computer Engineering from Chamran University in Iran in 2008.He received his MSc degree in Computer Engineering from Kouzestan Science and Research Branch, Islamic Azad University, in Iran in 2011. His research interests include image processing in License Plate Detection.

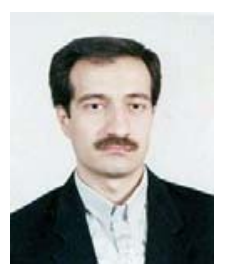

Reza Javidan received the B.Sc degree in Computer Engineering (hardware) from Isfahan University in 1993. He received M.Sc. and Ph.D. degree in Computer Science and Engineering (Artificial Intelligence) from Shiraz University in 1996 and 2007, respectively. He currently is an assistant professor and works as a lecturer in university.

He has more than 30 papers that were published in different national and international conferences and Journals. He also published a book about sonar systems.

Dr. Javidan major research interests include Pattern Recognition, Image Processing, Artificial Intelligence, sonar systems and Computer Vision.

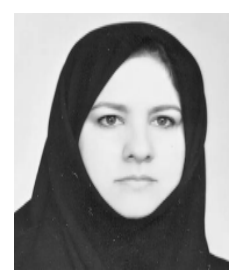

Manijeh Keshtgary is the head of Dept. of Computer Eng. \& IT, Shiraz University of Technology, Shiraz, Iran. She received her Master's degree in Electrical \& Computer Eng. from Colorado State University, CSU, Fort Collins, USA in 1993 and her $\mathrm{PhD}$ degree in Computer Eng. from Sharif University of technology in 2005. Dr.Keshtgary's research interests include MANET, Wireless Sensor Networks and GSM security issues. 